\title{
A Class OF FLAG TRANSITIVE PLANES
}

\author{
M. L. NARAYANA RAO
}

ABSTRACT. A class of translation affine planes of order $q^{2}$, where $q$ is a power of a prime $p \geqq 3$ is constructed. These planes have an interesting property that their collineation groups are flag transitive.

1. Introduction. Let $\pi$ be a finite affine plane of order $n$. A collineation group $G$ of $\pi$ is defined to be flag transitive on $\pi$ if $G$ is transitive on the incident point-line pairs, or flags, of $\pi$. A. Wagner [7] has shown that $\pi$ is a translation plane so that $n=p^{r}$ for some prime $p$ and for some integer $r>0$. D. A. Foulser [3], [4] has determined all flag transitive groups of finite affine planes. While determining the flag transitive groups Foulser remarks that the existence of non-Desarguesian flag transitive affine planes is still an open problem. However he constructs two flag transitive planes [4] of order 25 and shows that his two planes of order 25 and the near field plane of order 9 have flag transitive collineation groups. $C$. Hering [5] has constructed a plane of order 27 which has a flag transitive group. Recently the author [6] has constructed a plane of order 49 and has shown that it has a flag transitive group. The aim of this paper is to construct a class of non-Desarguesian affine translation planes of order $q^{2}$, where $q$ is a power of a prime $p \geqq 3$, which have flag transitive collineation groups.

2. Let $n=p^{f}$, where $p$ is a prime and $f$ is a positive integer. Let $V$ be a vector space of dimension $2 f$ over $\mathrm{GF}(p)$. Let $\left\{V_{i} \mid 0 \leqq i \leqq n\right\}$ be a set of $f$-dimensional subspaces of $V$. Let $\pi$ be an incidence structure defined with vectors of $V$ as points of $\pi$ and subspaces $V_{i}$ and their cosets (in the additive group of the vector space $V$ ) as lines of $\pi$ with inclusion as an incidence relation. It may be shown (Andre [1]) that the incidence structure $\pi$ is an affine (translation) plane if $V_{i} \cap V_{j}=\{0\}$, the subspace of $V$ consisting of the zero vector alone, for $i \neq j, 0 \leqq i \leqq n, 0 \leqq j \leqq n$. Further any linear transformation of $V$, which permutes the subspaces $V_{i}$ among themselves induces a collineation of $\pi$ fixing the point corresponding to the zero

Received by the editors May 6, 1971 and, in revised form, August 31, 1972.

AMS (MOS) subject classifications (1970). Primary 20B25, 50D35; Secondary 20N10, 05B25.

Key words and phrases. Projective planes, affine planes, flag transitivity, VeblenWedderburn systems. 
vector. It can be shown that $\pi$ is flag transitive if there exists a group of linear transformations of $V$ which permutes transitively the subspaces $V_{i}$ for $0 \leqq i \leqq n$.

3. Construction of a class of affine planes. Let $\alpha$ be a generator of the group of nonzero elements of $\mathrm{GF}\left(q^{4}\right)$, where $q$ is a power of a prime $p \geqq 3$. Let $\beta$ be the generator of the group of nonzero elements of GF $(q)$ given by $\beta=\alpha^{\left(q^{2}+1\right)(q+1)}$. Throughout this paper we use $d$ in place of the number $(q+1)$. Since the element $\alpha^{d}$ lies outside $\operatorname{GF}\left(q^{2}\right)$, it satisfies an equation

$$
f(x)=x^{4}+a_{3} x^{3}+a_{2} x^{2}+a_{1} x+a_{0},
$$

where the coefficients $a_{i}$ are from $\operatorname{GF}(q)$ and the polynomial $f(x)$ is irreducible in $\operatorname{GF}(q)$. Using the relations between the roots and the coefficients of equation (3.1) one may obtain the following:

$$
\begin{gathered}
a_{0}=\beta^{2}, \\
a_{1}=\beta a_{3}, \\
a_{3} \neq 0, \\
a_{2}+2 \beta+\left(\beta \alpha^{-d}+\alpha^{d}\right) a_{3}+\left(\alpha^{d}-\beta \alpha^{-d}\right)^{2}=0, \\
a_{3}+\left(\alpha^{d}+\beta \alpha^{-d}\right)+\left(\alpha^{d}+\beta \alpha^{-d}\right)^{q}=0, \\
a_{2}=2 \beta+\left(\beta \alpha^{-d}+\alpha^{d}\right)^{d} .
\end{gathered}
$$

The relations (3.2), (3.3) and (3.7) are easy to verify. Using (3.2) and (3.3) in the relation

$$
\alpha^{4 d}+a_{3} \alpha^{3 d}+a_{2} \alpha^{2 d}+a_{1} \alpha^{d}+a_{0}=0
$$

we obtain (3.5). The relation (3.6) is a consequence of (3.5) and (3.7). To prove (3.4) let us suppose that $a_{3}=0$. Then (3.8) becomes

$$
\alpha^{4 d}+a_{2} \alpha^{2 d}+\beta^{2}=0 .
$$

The relation (3.8)' implies that $\alpha^{2 d}$ satisfies a quadratic in GF( $q$ ), a contradiction since $\alpha^{2 d}$ does not belong to $\mathrm{GF}\left(q^{2}\right)$. Hence $a_{3} \neq 0$.

Lemma 3.1. Let $u=\left(a_{2}+2 \beta\right) a_{3}^{-1}+\beta \alpha^{-d}+\alpha^{d}$. Then $u \in \mathrm{GF}\left(q^{2}\right)$ and is not a square in $\mathrm{GF}\left(q^{2}\right)$. Consequently it may be expressed as $u=\alpha^{s\left(q^{2}+1\right)}$, where $s$ is a certain odd integer.

Proof. From the relation

$$
\left(a+b \alpha^{d}\right)^{q^{2}+1}=a^{2}+b^{2} \beta+a b\left(\beta \alpha^{-d}+\alpha^{d}\right),
$$

where $a, b \in \mathrm{GF}(q)$ and $a \neq 0 \neq b$, we obtain that $\left(\beta \alpha^{-d}+\alpha^{d}\right)$ is an element in $\operatorname{GF}\left(q^{2}\right)$. Consequently $u \in \operatorname{GF}\left(q^{2}\right)$. Suppose that $u$ is a square in $\operatorname{GF}\left(q^{2}\right)$. 
The relation (3.5) may now be written as

$$
\left(\alpha^{d}-\beta \alpha^{-d}\right)^{2}=-a_{3} u .
$$

Since any element of $\operatorname{GF}(q)$ is a square in $\operatorname{GF}\left(q^{2}\right)$, we obtain from (3.10) that $\left(\alpha^{d}-\beta \alpha^{-d}\right)^{2}$ is a square in $\operatorname{GF}\left(q^{2}\right)$ and consequently $\left(\alpha^{d}-\beta \alpha^{-d}\right) \in$ $\mathrm{GF}\left(q^{2}\right)$. This together with the fact that $\left(\beta \alpha^{-d}+\alpha^{d}\right) \in \mathrm{GF}\left(q^{2}\right)$ leads to a contradiction that $\alpha^{d} \in \mathrm{GF}\left(q^{2}\right)$. Thus $u \in \mathrm{GF}\left(q^{2}\right)$ and $u$ is not a square in $\operatorname{GF}\left(q^{2}\right)$. Since $u$ is not a square in $\operatorname{GF}\left(q^{2}\right), u$ may be expressed as $u=\alpha^{s\left(q^{2}+1\right)}$ where $s$ is a certain odd integer.

LeMmA 3.2. Let $v=\left(a_{2}+2 \beta\right)^{2} a_{3}^{-2}-4 \beta$. Then $v$ is not a square in $\operatorname{GF}(q)$.

Proof. Let $\left(a_{2}+2 \beta\right) a_{3}^{-1}=g,\left(\beta \alpha^{-d}+\alpha^{d}\right)=h$. We obtain from Lemma 3.1 that $\alpha^{s\left(q^{2}+1\right)}=(g+h)$ and therefore $(g+h)^{d}=\beta^{s}$ is not a square in $\mathrm{GF}(q)$, since $s$ is an odd integer. However, using the relations (3.6) and (3.7) we obtain that

$$
\begin{aligned}
(g+h)^{d} & =\left(g+h^{q}\right)(g+h) \\
& =g^{2}+g\left(h^{q}+h\right)+h^{d} \\
& =g^{2}+a_{2}-2 \beta+g\left(h^{q}+h\right) \\
& =g^{2}-4 \beta+g\left(a_{3}+h+h^{q}\right) \\
& =g^{2}-4 \beta .
\end{aligned}
$$

Hence the lemma.

Let $V_{0}$ be the vector space over $\operatorname{GF}(q)$ defined by the basis $\left\{1, \alpha^{d}\right\}$. Let $v$ and $\delta$ be linear transformations of $\mathrm{GF}\left(q^{4}\right)$ defined by

$$
\nu: x \rightarrow x \alpha^{2 d} \text { and } \delta: x \rightarrow x^{q} \alpha^{k}
$$

with $k \equiv s(\bmod d)$, where $s$ is the odd integer of Lemma 3.1. Let $\pi$ be the incidence structure whose points are the vectors of $V=\mathrm{GF}\left(q^{4}\right)$ and whose lines are the images of $V_{0}$ under the group $H=\langle\nu, \delta\rangle$ of linear transformations and their cosets in the additive group of $\operatorname{GF}\left(q^{4}\right)$, with inclusion as an incidence relation.

THEOREM 3.1. The incidence structure $\pi$ is a non-Desarguesian affine translation plane. Further the group $H$ of linear transformations induces a group of collineations of $\pi$ which fixes the origin and permutes the lines through the origin transitively.

In the course of the proof of Theorem 3.1 we need the following two lemmas.

Let $0 \neq x=a+b \alpha^{d}$ and $y=\left(b+\beta^{-1} a \alpha^{d}\right)$ be elements from $V_{0}$, where $a, b \in \mathrm{GF}(q)$. From the relation

$$
\left(x y^{-1}\right)^{q^{2}+1}=\beta
$$


we obtain that

$$
x y^{-1}=\alpha^{d+t\left(q^{2}-1\right)}=\alpha^{k_{x} d}
$$

for some integer $t$ and therefore $k_{x}$ is an odd integer, a function of $x$.

LEMMA 3.3. Let $0 \neq x=a+b \alpha^{d}, y=\left(b+\beta^{-1} a \alpha^{d}\right)$ and $z$ be elements of $V_{0}$ where $a, b \in \mathrm{GF}(q)$ and $x z^{-1} \notin \mathrm{GF}(q)$. Then $x=z \alpha^{c d}$ for some integer $c$ if and only if (i) $z=l y$ for some $l \in \mathrm{GF}(q)$ and (ii) $l \alpha^{c d}=\alpha^{k_{x} d}$. Further if $x=$ $z \alpha^{c d}$, then $c$ is an odd integer.

Proof. Obviously (i) and (ii) imply that $x=z \alpha^{c d}$ for some integer $c$ and $z=e+f \alpha^{d}$. Suppose that $x=z \alpha^{c d}$ for some integer $c$ and $z=e+f \alpha^{d}$. Obviously $z \neq 0$. From the relation $x^{q^{2}+1}=z^{q^{2}+1} \beta^{c}$ we obtain, after using the fact that $1, \alpha^{-d}, \alpha^{d}$ are linearly independent over $\mathrm{GF}(q)$,

$$
\begin{aligned}
a b & =e f \beta^{c}, \\
a^{2}+b^{2} \beta & =\left(e^{2}+f^{2} \beta\right) \beta^{c} .
\end{aligned}
$$

Eliminating $\beta^{c}$ from (3.13) and (3.14) we have

$$
(\beta b f-a e)(b e-a f)=0 .
$$

Since $x z^{-1} \notin \mathrm{GF}(q), b e-a f \neq 0$. We therefore have that $\beta b f-a e=0$ from which we obtain that $z=l\left(b+\beta^{-1} a \alpha^{d}\right)$ for some $l \in \mathrm{GF}(q)$. The condition (ii) now follows easily. Since $l \alpha^{c d}=\alpha^{k x^{d}}$, where $k_{x}$ is an odd integer, we have that $c$ also is an odd integer.

LeMmA 3.4. Let $M=\left\{x y \alpha^{l d} \mid x, y \in V_{0}, x \neq 0 \neq y, l\right.$ an integer $\}$. Then $\alpha^{m} \notin M$ where $m \equiv s(\bmod d)$ and $\alpha^{s\left(q^{2}+1\right)}=u$ of Lemma 3.1.

Proof. Let $x=a+b \alpha^{d}$ and $y=e+f \alpha^{d}$, where $a, b, e, f \in \mathrm{GF}(q)$. Suppose that $x y \alpha^{l d}=\alpha^{s+t d}$ for some integers $l$ and $t$. Then

$$
(x y)^{q^{2}+1} \beta^{l-t}=u
$$

using the relations (3.2), (3.3) and (3.8) in (3.16) we obtain

$$
\begin{aligned}
& \beta^{-t+l}\left((\beta b f+a e)^{2}+\beta\left(b^{2} e^{2}+a^{2} f^{2}\right)-a b e f a_{2}\right) \\
& +\beta^{l-t}\left((\beta b f+a e)(b e+a f)-a b e f a_{3}\right)\left(\beta \alpha^{-d}+\alpha^{d}\right) \\
& =\left(a_{2}+2 \beta\right) a_{3}^{-1}+\beta \alpha^{-d}+\alpha^{d} .
\end{aligned}
$$

Since 1 and $\left(\beta \alpha^{-d}+\alpha^{d}\right)$ are linearly independent over $\operatorname{GF}(q)$ we obtain, from (3.17),

$$
\begin{gathered}
\beta^{l-t}\left((\beta b f+a e)^{2}+\beta\left(b^{2} e^{2}+a^{2} f^{2}\right)-a b e f a_{2}\right)=\left(a_{2}+2 \beta\right) a_{3}^{-1} \\
\beta^{l-t}\left((\beta b f+a e)(b e+a f)-a b e f a_{3}\right)=1
\end{gathered}
$$


Eliminating $\beta^{l-t}$ from (3.18) and (3.19) we obtain

$$
\begin{aligned}
(\beta b f+a e)^{2}- & \left(a_{2}+2 \beta\right) a_{3}^{-1}(\beta b f+a e)(b e+a f)+\beta(b e+a f)^{2} \\
& =0 .
\end{aligned}
$$

Suppose $b e+a f=0$. Then $\beta b f+a e=0$. If we further suppose that any one of $a, b, e, f$ vanishes, then we obtain that either $x=0$ or $y=0$, contrary to the hypothesis. Thus in case $b e+a f=0$, we have $a \neq 0 \neq b, e \neq 0 \neq f$. Eliminating $a$ and $b$ from $b e+a f=0$ and $\beta b f+a e=0$ we obtain $\beta=e^{2} f^{-2}$, a square in $\mathrm{GF}(q)$, a contradiction. Thus $b e+a f \neq 0$. Equation (3.20) may now be written as

$$
w^{2}-\left(a_{2}+2 \beta\right) a_{3}^{-1} w+\beta=0,
$$

where $w=(\beta b f+a e)(b e+a f)^{-1}$. Since $\left(\left(a_{2}+2 \beta\right)^{2} a_{3}^{-2}-4 \beta\right)$ is not a square in $\mathrm{GF}(q)$, the relation (3.21) leads to a contradiction that $w$ satisfies a quadratic irreducible in $\mathrm{GF}(q)$. From this contradiction we infer the truth of the lemma.

Proof of Theorem 3.1. Let $V_{i}$ be the vector space over $\operatorname{GF}(q)$ generated by $\left\{\alpha^{2 i d}, \alpha^{(2 i+1) d}\right\}$ for $0 \leqq i \leqq\left(q^{2}-1\right) / 2$. Obviously $V_{i}$ is the image of $V_{0}$ under the linear transformation $v^{i}$ of $\mathrm{GF}\left(q^{4}\right)$. Let $U_{i}$ be the vector space over $\mathrm{GF}(q)$ generated by $\left\{\alpha^{k+d(q+2 i)}, \alpha^{k+2 i d}\right\}$ for $0 \leqq i \leqq\left(q^{2}-1\right) / 2$. As before it may be shown that $U_{0}$ is the image of $V_{0}$ under the linear transformation $\delta$ and $U_{i}$ is the image of $U_{0}$ under the linear transformation $\nu^{i}$ of $\operatorname{GF}\left(q^{4}\right)$. Further from the relation

$$
\left(a \alpha^{2 i d}+b \alpha^{(2 i+1) d}\right) \delta=\alpha^{2 i q d}\left(a \alpha^{k}+b \alpha^{k+q d}\right),
$$

where $a, b \in \mathrm{GF}(q)$, we obtain that $V_{i} \delta=U_{j}$ where $i q \equiv j\left(\bmod \left(q^{2}+1\right) / 2\right)$. Similarly from the relation

$$
\left(a \alpha^{k+2 i d}+b \alpha^{k+(2 i+q) d}\right) \delta=\alpha^{(2 i q d)+(k-1) d}\left(b \beta+a \alpha^{d}\right)
$$

we obtain that $U_{i} \delta=V_{j}$, where $i q+(k-1) / 2 \equiv j\left(\bmod \left(q^{2+1}\right) / 2\right)$. Thus the set $P$ of images of $V_{0}$ under the group $H=\langle\nu, \delta\rangle$ of linear transformations of $\mathrm{GF}\left(q^{4}\right)$ consists of $V_{i}$ and $U_{j}$ for $0 \leqq i \leqq\left(q^{2}-1\right) / 2$ and $0 \leqq j \leqq\left(q^{2}-1\right) / 2$ and $H$ is transitive on the set $P$. We may now conclude that, if $\pi$ is an affine plane, then $H$ induces a collineation group which fixes the origin and permutes the lines through the origin transitively.

To prove that $\pi$ is an affine plane we have to show that $X_{i} \cap Y_{j}=$ $\{0\}$ if $X_{i} \neq Y_{j}$ and $X=U$ or $V$ and $Y=U$ or $V, 0 \leqq i \leqq\left(q^{2}-1\right) / 2,0 \leqq j \leqq$ $\left(q^{2}-1\right) / 2$. Without loss of generality we may suppose $i \leqq j$. Then from the relations $\left(X_{i} \cap Y_{j}\right) v^{-i}=X_{0} \cap Y_{j-i}$ and $\left(U_{i} \cap U_{j}\right) \delta^{-1}=V_{0} \cap V_{j-i}$ we have that

$$
\begin{array}{ll}
X_{i} \cap Y_{j}=\{0\} & \text { if and only if } X_{0} \cap Y_{j-i}=\{0\}, \\
U_{i} \cap U_{j}=\{0\} & \text { if and only if } V_{0} \cap V_{j-i}=\{0\} .
\end{array}
$$


In view of (3.22) and (3.23) it is enough if we show that

$$
\begin{array}{ll}
V_{0} \cap V_{i}=\{0\} & \text { for } 0 \leqq i \leqq\left(q^{2}-1\right) / 2, \\
V_{0} \cap U_{i}=\{0\} & \text { for } 0 \leqq i \leqq\left(q^{2}-1\right) / 2 .
\end{array}
$$

Obviously $V_{0}, V_{i}, U_{j}$ contain the zero vector. To prove (3.24) let us suppose $x \in V_{0}, y \in V_{i}$, with $x \neq 0 \neq y$ and $x=y$. Then there is a $z \in V_{0}$ such that $y=z \alpha^{2 i d}$ and consequently $x=z \alpha^{2 i d}$. This in view of Lemma 3.3 leads to a contradiction that $2 i$ is an odd integer, since $x z^{-1} \notin \mathrm{GF}(q)$. Suppose $0 \neq x \in V_{0}$ and $0 \neq y \in U_{i}$ and $x=y$. Then there is a $z \neq 0$ in $U_{0}$ such that $y=z \alpha^{2 i d}$. Let $z=\left(e+f \alpha^{q d}\right) \alpha^{k}$ where $e, f \in \mathrm{GF}(q)$. Then $e+f \alpha^{q d}=$ $\left(e+f \alpha^{d}\right)^{q}=\left(e+f \alpha^{d}\right)^{-1}\left(e+f \alpha^{d}\right)^{d}$. Let $e+f \alpha^{d}=\alpha^{t}$. Now $x=y$ implies that $x\left(e+f \alpha^{d}\right)=\alpha^{k+t d+2 i d}$ contrary to Lemma 3.4. Thus $\pi$ is an affine plane.

Obviously $A=\left\langle\alpha^{2 d}\right\rangle$ induces a group of collineations of $\pi$ and its order is $\left(q^{2}+1\right)(q-1) / 2$. Suppose $T$ is an odd prime which divides $\left(q^{4}-1\right)$ but does not divide $\left(p^{i}-1\right)$ for $0<i<4 r$ (such a prime exists in view of Corollary 2 , p. 358 of Artin [2]). Obviously $T$ is not a factor of $2(q+1)$. Otherwise we obtain a contradiction that $q^{2} \equiv 1(\bmod T)$. It then follows that $T$ is a factor of $\left(q^{2}+1\right)(q-1) / 2$, the order of $A$ and satisfies conditions (2) and (3) of Lemma 3.1 of Foulser [4]. We now claim that $V_{0}$ is not of the form $A\left(\mathrm{GF}\left(q^{2}\right)\right)$ for any $a \neq 0$ from $\mathrm{GF}\left(q^{4}\right)$. Suppose the contrary. Then if $b \neq 0$ and $b V_{0}$, it follows that $b^{-1} V_{0}=\mathrm{GF}\left(q^{2}\right)$ and it may be shown that it is not the case by taking $b=\alpha^{d}$ and noting that $\alpha^{-d} \notin \mathrm{GF}\left(q^{2}\right)$. We now invoke Lemma 6.1 of Foulser [4] to claim that $\pi$ is non-Desarguesian. This completes the proof of the theorem.

Classification of these planes into nonisomorphic classes will be discussed elsewhere.

\section{REFERENCES}

1. J. André, Über nicht-Desarguesche Ebenen mit transitiver Translationsgruppe, Math. Z. 60 (1954), 156-186. MR 16, 64.

2. E. Artin, The orders of linear groups, Comm. Pure Appl. Math. 8 (1955), 355-366. MR 17, 12.

3. D. A. Foulser, The flag-transitive collineation groups of the finite Desarguesian affine planes, Canad. J. Math. 16 (1964), 443-472. MR 29 \#3549.

4. - Solvable flag transitive affine groups, Math. Z. 86 (1964), 191-204. MR 30 \#1190.

5. C. Hering, Eine nicht-desarguesche zweifach transitive affine Ebene der Ordnung 27, Abh. Math. Sem. Univ. Hamburg 34 (1969/70), 203-208. MR 42 \#8390.

6. M. L. Narayana Rao, A flag transitive plane of order 49, Proc. Amer. Math. Soc. 32 (1972), 256-262.

7. A. Wagner, On finite affine line transitive planes, Math. Z. 87 (1965), 1-11. MR 30 \#2391.

Department of Mathematics, Osmania University, Hyderabad-7 (A.P.), India 